\title{
Adaptive Image Steganography by Reversible Data Hiding
}

\author{
Guangjie Liu ${ }^{1}$ Yuewei Dai ${ }^{1}$ Zhiquan Wang $^{1}$ Shiguo Lian ${ }^{2}$ \\ ${ }^{1}$ Automation School of Nanjing University of Sci.\& Tech. Nanjing 210094, China \\ ${ }^{2}$ France Telecom R\&D Beijing, Beijing 100006, China
}

\begin{abstract}
An adaptive steganographic scheme is proposed in this paper. The adaptive quantization embedder is introduced and employed by block-wised fashion. We also constructed contrast-correlation distortion metric to optimally choose quantization steps for image blocks to guarantee more data being embedded in busy areas. Different form existing methods, our scheme embeds the AQE parameters together with message bits into the cover image by difference expanding algorithm. Simulation experiments show the proposed scheme can provide a good trade-off between the perceptibility and the capacity.
\end{abstract}

Keywords: Steganography, Adaptive quantization embedder, Construct-correlation distortion, Difference expanding

\section{Introduction}

Imperceptibility and capacity are the two important properties of any steganographic schemes, the former ensures that the embedding is imperceptible (can not be detected by human eyes), and the latter indicates the efficiency of covert communication. There have been lots of techniques proposed to balance the trade-off between them. Wang et al. [6] developed a technique to hide secret data by LSB substitution and a genetic algorithm(GA). Chang et.al. [1] used dynamic programming strategy to replace GA in [6]. In [2], Chan et. al. proposed a simple LSB substitution with an optimal pixel adjustment process(OPAP). These techniques can all be concluded as LSB-like methods, which realized optimal data embedding in meanings of mean square error(MSE).

It is known for us that MSE is not a good metric for measuring the image degradation caused by information hiding, so the above methods cannot provide famous performance. Therefore lots of endeavors have been made for performance improve- ments. Wu [4] proposed a steganography method for images by pixel-value differencing, which divided the image into two-pixels block, and embedded information into the difference value of each two pixels. BPCS [5]divided the image into regions and performed complexity measurement using a binary complexity measure on the individual bit planes to embed data in these regions. In [8], Zhang proposed to convert data into a series of symbols in a notation system with multiple bases, and the specific bases were obtained by the degree of local variations of the neighboring pixels in the stego image. In [3], Yang and Lin proposed a base-oriented hiding algorithm, which classified each block of the host image according to the base value, and make data embedding according some predeterminative parameters. After reviewing them, we can find it without hard that different from LSB-like methods, they all adopt the adaption mechanism(i.e. different quantization steps for different pixels). Despite that the adaption mechanism can achieve good performance in experimental meanings, there is not a proper framework to tell us why those chosen quantization steps are suitable and how to obtain them.

In this paper, we propose a new steganographic method to hide data in gray images via adaptive quantization-embedder(AQE). In the scheme, we construct a new distortion metric called contrastcorrelation distortion, the message bits are embedded into image blocks via an optimally searched quantization step under a given distortion constraint. The remainder of the paper is organized as following. Section 2 introduces the principles of adaptive quantization-embedder. Section 3 discusses the new construct distortion metric. Section 4 discuss the application of reversible data hiding algorithm in our scheme. Section 5 details the implementation of the whole algorithm. Section 6 gives the simulation experiments. Section 7 concludes the whole paper. 


\section{Principles of AQE}

As we know, not all pixels in an image can tolerate the same amount of changes without causing noticeable distortion. According to the basic principle of HVS, the changes occurring in the smooth area can be easily noticed by human eyes, while busy or edged image regions can accommodate more hiding space under the same distortion restriction. So there must be some adaption mechanism to be adopted to catch the HVS characteristics of the cover image and provide more steganographic capacity and less embedding distortion.

\subsection{Definition of AQE}

Suppose the cover image $X$ to be composed of pixels such as $x_{1}, x_{2}, \ldots, x_{L}$, we can write the quantization-embedder(QE) with integer step being $q$ as:

$$
\begin{aligned}
& \Phi(x, q)=x-[x, q] \\
& x^{\prime}=\Phi(x, q)+m ; \\
& \Omega=\left\{x^{\prime}, x^{\prime}+q, x^{\prime}-q\right\} \\
& y=\operatorname{argmin}_{z \in \Omega}(\|z-x\|)
\end{aligned}
$$

Here $x$ belongs to $\left\{x_{1}, x_{2}, \ldots, x_{L}\right\}, \Phi(x, q)$ is the quantization function, $[x, q]$ denotes to calculate the residue of $x$ divided by $q$, and $m$ represents the integer between $[0, q-1]$ which may be converted from the message bits. Eq. (4) is used to find the optimal value which has the residue after dividing $q$ is equal to $m$. We can rewrite the whole process as $y=\Upsilon(x, q, m)$.

When the quantization step $q$ is fixed and independent on different pixels, we call the quantizatinembedder as fixed one. While, if $q$ changes with the corresponding pixel, the quantizationembedder are called adaptive one. In pixel-wised adaptive quantization-embedders, the step $q_{i}$ for each pixel is different, which means the most meticulous parameters choosing manner. But in most case it is unnecessary to chose different $q$ for different pixel. The more practical and efficient fashion is to choose one quantization step for a set of neighboring pixels, i.e. one block, because the pixels in one block may have the same HVS characteristics.

Comparing with pixel-wised AQE, block-wised AQE does not only hold less computation complexity, but also has the advantage to dispose quantization parameters, because the quantization step vector $Q$ has less dimension than that of pixelwised one. In this paper, we concentrate our attention on the block-wised adaptive quantization- embedder. Suppose that the cover image pixels are divided into blocks $b_{1}, b_{2}, \ldots b_{i}, \ldots, b_{N}$, which have the same size $n \times n$, the quantization step vector $Q$ is defined as $\left[q_{1}, q_{2}, \ldots, q_{N}\right]$. The steganography process can be described as

$$
Y=\Upsilon(X, Q, M)
$$

Here, $M=\left[\mu_{1}, \ldots, \mu_{i}, \ldots \mu_{n}\right], \quad \mu_{i}=$ $\left[m_{i 1}, \ldots, m_{i j}, \ldots, m_{i n^{2}}\right]$ and $m_{i j}$ is the integer falling in $\left[0, q_{i}-1\right]$.

The data extracting process can be described as

$$
M=Y-\Phi(Y, Q)
$$

the embedding message can be converted from the extracted $M$ according to the step vector $Q$.

\subsection{Parameters of AQE}

According to the extraction rule Eq.(6), for extracting the embedded message, the quantization step vector $Q$ must be known by the extractor, which is greatly different from the conditional methods with fixed quantization steps. So it becomes a key point to transmit the information of $Q$ from embedder to extractor.

There might be two kinds of methods competent for the mission. In the first one, the adaptive quantization steps are constructed according to the stego image itself like [8] and [3]. In [8], Zhang proposed use variation of three pixels, $p(i-1, j), p(i, j-1)$ and $p(i-1, j-1)$ of stegoimage to construct the base used for data hiding in pixel $p(i, j)$, it is easy to reconstruct the base according to stego image for extractor. In [3], Yang proposed to use the base value(BV) of one $3 \times 3$ block as the base to make message bit conversion and data hiding, and to check BV of stego block. If $\mathrm{BV}$ of stego block is not equal to that of cover block, Yang proposed to make some adjustment to make them equal or directly perform module substitution scheme.

The second method is to directly embed the parameters in images, and extract these parameters at receiver side before the message extracting. For example, we can use part of cover image pixels to carry the parameters information and others to carry the true secrete message bits, or use reversible data hiding manner to embed parameters information in the stego image after true message hiding. Of course, the former will cause the efficient capacity decreased, the latter will introduce the supererogatory distortion. But if the 
size of block is chosen appropriately,and some efficient compression algorithm aiming at parameters of AQE is employed, the payload of parameters will be economized markedly. So the capacity or distortion influence introduced by parameters embedding will be control to acceptable extent.

In this paper, we adopt the method to using reversible data hiding method to embed the parameters information into the stego image after message bit embedding. At extractor side, the parameters is extracted in advance and the stego image is restored, then the message bits are extracting according to the parameters from the restored image.

\subsection{Capacity}

If we define the capacity of steganography as the ratio between the bit numbers of embedded message and the host image, then the capacity can be depicted as Eq. (7) according to the discussions in 2.1 and 2.2 , we can deduce the capacity.

$$
C=\sum_{i=1}^{N}\left\lfloor\log _{2}\left(q_{i}\right) \cdot\left(n^{2}\right)\right\rfloor
$$

where, $\lfloor\cdot\rfloor$ is the function to get the largest integer that is less than ".". In data embedding process, for block $b_{i}$, if the embedder know the quantization step $q_{i}$, he just need read $\left\lfloor\log _{2}\left(q_{i}\right) \cdot\left(n^{2}\right)\right\rfloor$ from the message bit sequence, and convert them into $n^{2}-$ 1 integers between $\left[0, q_{i}-1\right]$ from binary system to $q_{i}$-system. The data extracting is the adverse manipulation.

Obviously, the capacity is the function of $Q$, so the capacity can be written as $C(Q)$. And the larger the components of $Q$ is, the higher the achievable capacity is. While increase the larger quantization step $q_{i}$ will also cause the corresponding distortion enlarged. So for any AQE scheme, it is essential problem how to choose the parameters of AQE, i.e. $Q$. The parameters acquirement also demands more applicable distortion metric, because if only MSE is used, the optimality of quantization parameters becomes meaningless, which also implies the adopted distortion metric should be adjacent to HVS.

\section{Contrast-correlation distor- tion}

In the most works to evaluate the performance of a steganographic technique, MSE and PSNR are always the requisite. However, they are not very accurate perceptually because of not matching well with the HVS characteristics. In [9], Wang and Bovik presented a new numerical measure for gray scale images, called the universal image quality index, which is defined as

$$
\theta=\frac{4 \sigma_{x y} \mu_{x} \mu_{y}}{\left(\sigma_{x}^{2}+\sigma_{y}^{2}\right)\left(\mu_{x}^{2} \mu_{y}^{2}\right)}
$$

where $x_{i}, y_{i}$ represent the original and disorted pixels respectively, $\mu_{x}=\frac{1}{n} \sum_{i=1}^{n} x_{i}, \mu_{y}=\frac{1}{n} \sum_{i=1}^{n} y_{i}$ $, \sigma_{x}^{2}=\frac{1}{n-1} \sum_{i=1}^{n} x_{i}-\mu_{x}, \sigma_{y}^{2}=\frac{1}{n-1} \sum_{i=1}^{n^{n}} y_{i}-\mu_{x}$ and $\sigma_{x y}=\frac{1}{n-1} \sum_{i=1}^{n}\left(x_{i}-\mu_{x}\right)\left(y_{i}-\mu_{y}\right)$. As described in [9], this quality index models the distortion as a combination of three factors: the luminance, contrast and correlation comparison, such as $\frac{2 \mu_{x} \mu_{y}}{\mu_{x}^{2}+\mu_{y}^{2}}, \frac{2 \sigma_{x} \sigma_{y}}{\sigma_{x}^{2}+\sigma_{y}^{2}}$ and $\frac{\sigma_{x y}}{\sigma_{x} \sigma_{y}}$.

The quality index $\theta$ is applied to a gray image using a sliding window with window size of $8 \times 8$. The index is computed for each window, leading to a quality map of the image The overall quality index is the average of all $\theta$ value in the quality map. Because $\theta$ produces unstable results when either $\mu_{x}^{2}+\mu_{y}^{2}$ or $\sigma_{x}^{2}+\sigma_{y}^{2}$ is close to zero. To avoid this problem the measure has been generalized to the structural similarity index(SSIM) [10].

Considering the distortion measurement between cover image block and stego block after AQE steganography, when the embedded message bits are encrypted or compressed before hiding, the binary message bit obeys uniform distribution on $\{0,1\}$. For an arbitrary block $b$, suppose the used quantization step to be $q$, the converted integers $m$ must obey the uniform distribution on $\{0,1, \ldots, q-1\}$. Reviewing Eq.(2), It is also true that the residues $m$ obey uniform distribution on $\{0,1, \ldots, q-1\}$ for natural images when $q$ is not too large. Base on the above analysis, the luminance comparison of AQE is not important, and note that the computation of contrast and correlation comparison can reflect the impact of luminance change, so only the contrast and correlation comparison are taken into consideration in this paper.

Between the contrast and correlation comparison, the former gives the whole measurement of noise introduced by data embedding, and the latter summarized the correlation distortion on each pixel. For evaluate the distortion of data embedding subtly, we prefer to emphasize the correlation comparison and combine the two by the below 
equation.

$$
\varphi(x, y)=\left(1-\frac{2 \sigma_{x} \sigma_{y}+C}{\sigma_{x}^{2}+\sigma_{y}^{2}+C}\right) \cdot\left(\frac{1+\frac{\sigma_{x y}+C}{\sigma_{x} \sigma_{y}+C}}{2}\right)^{2}
$$

, where $x, y$ denotes the cover and stego image block, and $\mathrm{C}$ is the constants equal to $(K R)^{2}$ with $R$ the dynamic range of the pixel value( 255 for 8-bit gray images) and $K \ll 1 . C$ is introduced to get more stable results like [10]. The first term of Eq. (9) is contrast distortion, which falls into the range $[0,1]$ with 0 stands for no distortion and 1 standing for largest distortion. Because $\frac{\sigma_{x y}}{\sigma_{x} \sigma_{y}}$ belong to the set $[-1,1]$, so the transform from correlation comparison to distortion is different form the contrast one, and we assign 2 for the exponent of correlation distortion to emphasize its significance. we named the combined distortion defined by Eq.(9) as contrast-correlation distortion(CCD), which always falls in the range $[0,1]$. The smaller the value of CCD is, the less distortion introduced by data embedding is.

\section{Reversible data hiding method}

Assuming that the size of block is equal to $n \times n$, the size of cover image is equal to $W \times H$, and the maximum value of quantization step does not exceed $q_{\max }$, we can obtain the length of the parameter $\mathcal{L}$ is nearly equal to:

$$
\mathcal{L}=\left\lfloor\frac{W}{n}\right\rfloor \cdot\left\lfloor\frac{H}{n}\right\rfloor \cdot\left\lceil\log _{2}\left(q_{\max }\right)\right\rceil
$$

Our experiments show that through the compression algorithm, ZLIB, provide by JAVA.UTIL.ZIP.DEFLATEROUTPUTSTREAM, this data length can be reduced to about $40 \%$, i.e. the actual length is about $40 \%\left\lfloor\frac{W}{n}\right\rfloor \cdot\left\lfloor\frac{H}{n}\right\rfloor \cdot\left\lceil\log _{2}\left(q_{\max }\right)\right\rceil$. For example, if the block size is equal to $5 \times 5$, the image size is equal to $512 \times 512$, the $\max$ allowed quantization step is equal to $32, \mathcal{L}$ is about 20808 . so we need a high capacity reversible data hiding method which can provide at least $0.079 \mathrm{bbp}$ (bits per pixel). For a general case, if we suppose the compression ratio is $\rho$, the demanded capacity $\mathcal{C}$ provided by the reversible data hiding algorithm can be expressed as:

$$
\mathcal{C} \approx(1-\rho) \frac{\log _{2}\left(q_{\max }\right)}{n^{2}}
$$

Fortunately, Tian presented a promising high capacity reversible data embedding in [11]. In the algorithm, two techniques are employed, i.e., difference expansion and generalized least significant bit embedding, to achieve a very high embedding capacity, while keep the distortion low. The main idea of this technique is described below.

For a pair of pixel value $x$ and $y$, the algorithm first computes the integer average $l$ and difference $h$ of $x$ and $y$, where $h=x-y$. Then $h$ is modified to $h^{\prime}=2 \times h+b$, where $h^{\prime}$ denotes the expanded difference, which explains the term of Difference Expansion. Finally the new $x$ and $y$, denoted by $x^{\prime}$ and $y^{\prime}$, respectively, are calculate based on the new difference value $h^{\prime}$ and the original integer average value $l$. In this way, the stego image is obtained. To avoid over/underflow, the algorithm only embeds data into the pixel pairs that shall not lead to over/underflow. Therefore, a two-dimensional location map is compressed by JBIG2 and embedded as overhead.

In DE reversible method, Tian create four disjoint sets of difference values, $\mathrm{EZ}, \mathrm{EN}, \mathrm{CN}$ and $\mathrm{NC}$ :

- EZ: contains all expandable $h=0$ and expandable $h=-1$.

- EN: contains all expandable $h \notin E Z$.

- $\mathrm{CN}$ : contains all changeable $h \notin E Z \cup E N$.

- NC: contains all nonchangeable $h$.

and the set EN are partitioned by a threshold $T$ according to:

$$
\begin{aligned}
& E N 1=\{h \in E N:|h| \leq T\} \\
& E N 2=\{h \in E N:|h|>T\}
\end{aligned}
$$

Assume the total number of 1 and -2 in EN2U $C N$ is $\mathcal{N}$, then the capacity of Tian's DE method is about:

$$
\mathcal{C} \leq \frac{|E Z|+|E N 1|+\mathcal{N}-\mathcal{Z}}{W H}
$$

where $|\cdot|$ is the cardinality of a set, $\mathcal{Z}$ is the length of compressed binary location map. According to Eq. (11), we can control the threshold $T$ to satisfy the below equation.

$$
(1-\rho) \frac{\log _{2}\left(q_{\max }\right)}{n^{2}} \leq \frac{|E Z|+|E N 1|+\mathcal{N}-\mathcal{Z}}{W H}
$$

\section{The whole AQE algorithm}

In Section 2 and Section 3, we give the basic concepts of the AQE and analysis the capacity and proposed a new distortion metric. In Section 4, we discuss the reversible data hiding method used in our paper. In this section, we will give the whole data embedding and extracting algorithm. 
Table 1: Experimental results of four standard test images

\begin{tabular}{c|cc|cc|cc|cc}
\hline \hline Cover image & \multicolumn{2}{|c|}{ Capacity } & \multicolumn{2}{c|}{ wPNSR $(\mathrm{dB})$} & \multicolumn{2}{c|}{ Waston Metrics } & \multicolumn{2}{c}{ SSIM } \\
\cline { 2 - 9 } & $\varepsilon=0.01$ & $\varepsilon=0.03$ & $\varepsilon=0.01$ & $\varepsilon=0.03$ & $\varepsilon=0.01$ & $\varepsilon=0.03$ & $\varepsilon=0.01$ & $\varepsilon=0.03$ \\
\hline Lena & 0.198 & 0.302 & 44.3 & 40.1 & 0.0490 & 0.0677 & 0.9713 & 0.9511 \\
Baboon & 0.313 & 0.427 & 43.7 & 40.6 & 0.0612 & 0.0936 & 0.9704 & 0.9545 \\
Peppers & 0.199 & 0.311 & 43.5 & 39.8 & 0.0530 & 0.0751 & 0.9685 & 0.9518 \\
Boat & 0.239 & 0.344 & 43.6 & 39.1 & 0.0507 & 0.0820 & 0.9719 & 0.9513 \\
\hline \hline
\end{tabular}

\subsection{Encoding algorithm}

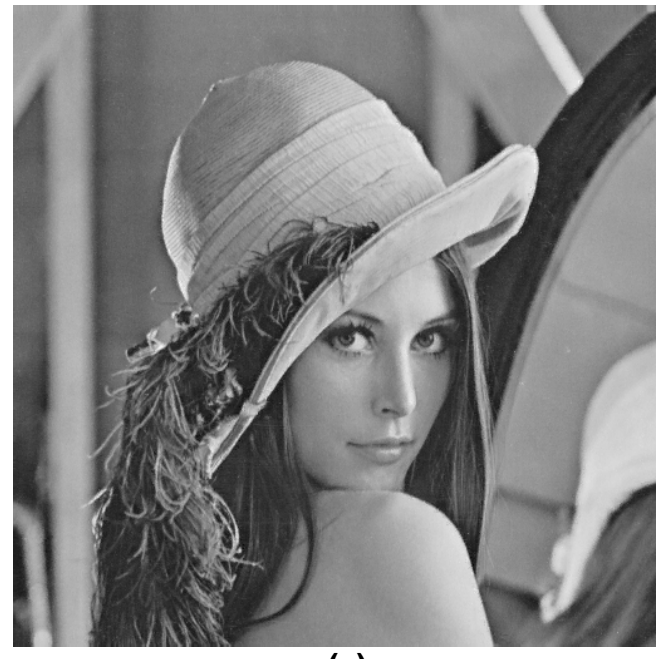

(a)

(a) stego image of Lena with $\varepsilon=0.02$

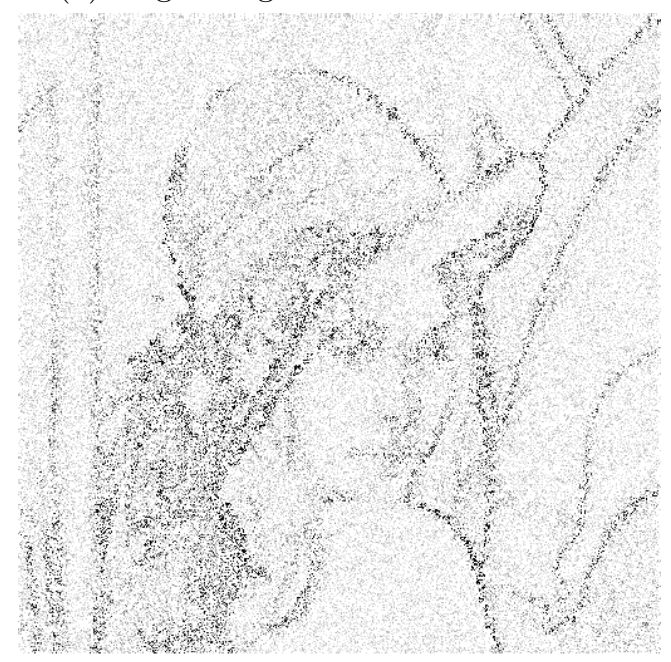

(b)

(b) Enhanced error image of Lena

Fig. 1: Experimental results of Lena
Initialization: Read a finite binary message bit sequence $M$, divide the $W \times H$ cover image into $V\left(V=\frac{W H}{n^{2}}\right)$ nonoverlapping blocks $\left\{b_{1}, b_{2}, \ldots, b_{V}\right\}$, each with size $n \times n$, set the allowed distortion index be $\varepsilon$, let $\kappa=0, Q=$ [], set the block reading order using the stego-key $K$.

Message Embedding: Deal with block according to the assigned order. For the current block $b_{i}$, allocate the quantization step candidate range $\Xi$ according to the below equation, $\langle A, B\rangle$ stands for all integers falling in the range $[\mathrm{A}, \mathrm{B}]$.

$$
\Xi=\left\langle\min \left(\alpha \sigma_{b_{i}}, 30\right)+2, \max \left(\beta \sigma_{b_{i}}, 2\right)\right\rangle
$$

Here, $\sigma_{b_{i}}$ is the standard deviation of block $b_{i}, \alpha$ and $\beta$ are two parameters to control the size of candidate range, we choose $\alpha=1.5$ and $\beta=0.2$ in the propose algorithm. Compute the below equations to obtain the optimal quantization step $q^{*}$.

$$
\begin{aligned}
& q^{*}=\max \left(\arg \max _{\varphi\left(b_{i}^{\prime}, b_{i}\right) \leq \varepsilon}(q), \min (\Xi)\right)(15) \\
& b_{i}^{\prime}=\Upsilon\left(b_{i}, q, \mu\right) \\
& \mu=\Psi\left(M\left(\kappa, \kappa+\left\lfloor n^{2} \log _{2}(q)\right\rfloor\right), q\right)
\end{aligned}
$$

Here, $\Psi\left(M\left(\kappa, \kappa+\left\lfloor n^{2} \log _{2}(q)\right\rfloor\right), q\right)$ means to convert the binary sequence $\left.M\left(\kappa, \kappa+\left\lfloor n^{2} \log _{2}(q)\right\rfloor\right)\right)$ into $n^{2} q$-system integers.

After Message Embedding in block $b_{i}$, update $\kappa$ by $\kappa \leftarrow \kappa+\left\lfloor n^{2} \log _{2}(q)\right\rfloor$, add $q^{*}$ to the end of $Q$, move to the next block, and repeat the same embedding process until all blocks are occupied or message bits are embedded completely. In this paper, we just discuss the step situation that all blocks are occupied.

Parameters Embedding: Before embedding parameters, we first use DPCM to encode $Q$ and make further compresion by ZLIB(JAVA.UTIL.ZIP.DEFLATEROUTPUTSTREAM)to get the ultimate compressed parameters $G$ with size equal to $U$ bits. According to $U$ and Eq. (13) to choose proper threshold and make reversible data hiding based on difference expanding. 


\subsection{Decoding algorithm}

The decoding part is much simpler than the encoding part. The extractor just need first extract AQE parameters $G$, and perform corresponding decoding to get the true quantization step vector $Q$. Once $\mathrm{Q}$ is obtained, the decoding of message bits is very simple according to Eq.(6). Through the corresponding conversion from $q_{i}$-system to binary system, all message bits can be resumed immediately.

\section{Experimental Simulation}

Using a $512 \times 512$ 8-bit gray image "lena" as the cover image and choosing $\varepsilon=0.02, n=5$, a total of $5.57 \times 10^{5}$ secret bits are embedded, so the capacity is equal to 0.25 . The stego images are shown in Fig.1 (a). Also shown is the error image, which has been enhanced by a 35-time gray-level stretch for the purpose of display. The similar experimental results are shown in Fig.2 for the cover image baboon. We can see that the modification were mainly in busy areas and on edges, which means less perceptual distortion is achieved.

Three quality metrics are used to measure the distortion induced by data embedding: wPNSR, the Watson metric, and SSIM[10]. The wPNSR and Watson metric are all designed in [7] by using characteristics of HVS and measure the total perceptual error. Four standard test images, lena, baboon, peppers and boat are used to make the second experiment. Table 1 lists the value of capcity, wPSNR, Waston metrics and SSIM under $\varepsilon=0.01$ and $\varepsilon=0.03$.

It can be seen from Table. 1 that the images with more edges and textures can carry more information than the flat one, and given the larger $\varepsilon$, the AQE can provides more space to carry message bits. So the propose method can be adaptive with the different image region and different image.

\section{Conclusions}

In this paper, we gave the basic principles of AQE and introduced two kinds of methods to deal with AQE parameters. In the proposed steganographic scheme, we suggested to embed the AQE parameters together with secret message bits by the difference expanding algorithm, and use the new constructed contrast-correlation distortion metric to control the block distortion. Because more data are embedded in textured areas and on edges that can tolerate more modifications, a high capacity is

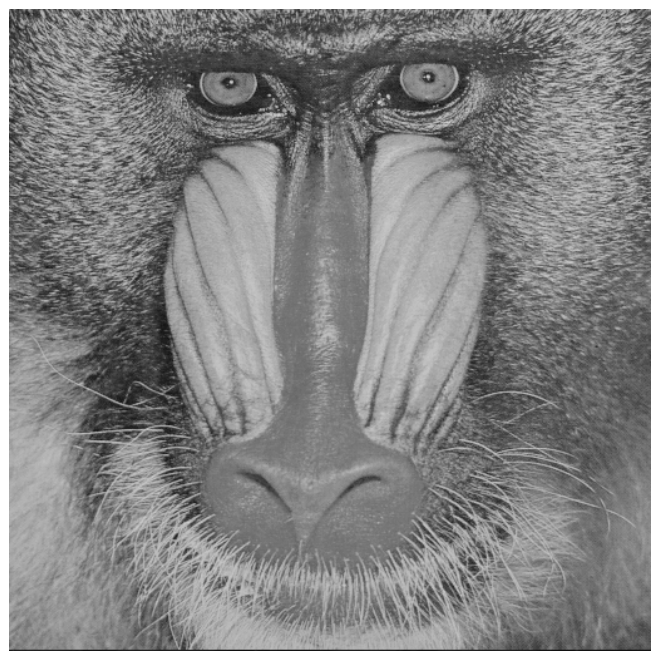

(a) stego image of Baboon with $\varepsilon=0.02$

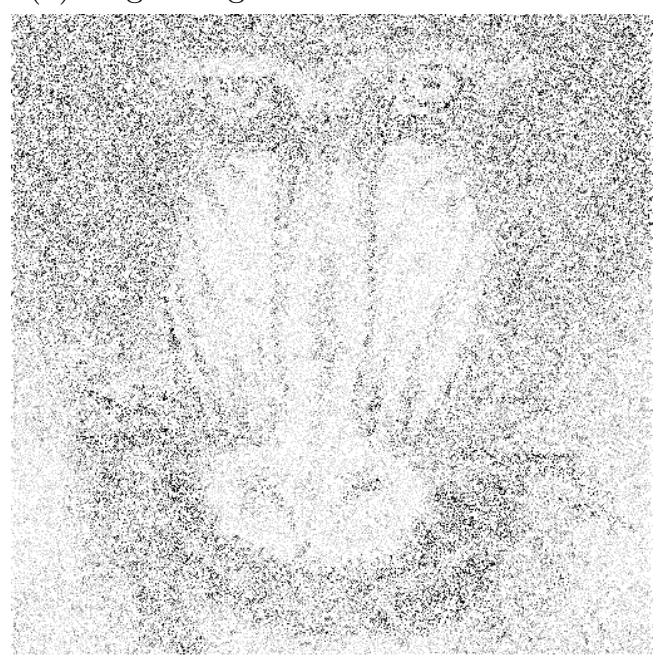

(b) Enhanced error image of Baboon

Fig. 2: Experimental results of Baboon

obtained while maintaining a good visual performance. Simulation experiments show the proposed scheme can provide a good trade-off between the perceptibility and the capacity.

\section{References}

[1] C.C. Chang, J.Y. Hsiao, C.S. Chan. Finding optimal leastsignificant- bit substitution in image hiding by dynamic programming strategy. Pattern Recognition, 36(7):1583-1585,Elsevier, 2003.

[2] C.K. Chan. L.M. Cheng. Hiding data in images by simple lsb substitution. Pattern Recognition, 37(3):469-474, Elsevier, 2004.

[3] C.Y. Yang, J.C. Lin. Image hiding by 
base-oriented algorithm. Optical Engineering, 45(11):117001.1-117001.10, SPIE, 2006.

[4] D.C. Wu. A steganographic method for images by pixelvalue differencing. Pattern Recognition Letters, 24(9- 10):1613-1626, 2003.

[5] R. Eason. A tutorial on bpcs steganography and its applications. Proceedings of IEEE PacificRimWorkshop on Digital Steganography, 183̃1, 2003.

[6] R.Z. Wang, C.F. Lin, J.C. Lin. Image hiding by optimal LSB substitution and genetic algorithm. Pattern Recognition, 34(3):671-683, 2001.

[7] S. Voloshynovskiy, S. Pereira, V. Iquise, T. Pun. Attack modelling: towards a second generation watermarking benchmark. Signal Processing, 81(6):1177-1214, 2001.

[8] X.P. Zhang, S.Z. Wang. Steganography using multiple-base notational system and human vision sensitivity. IEEE Signal Processing Letters, 12(1):67-70, 2005.

[9] Z. Wang, A.C. Bovik. A universal image quality index. IEEE Signal Processing Letters, 9(3):8184, Mar. 2002.

[10] Z. Wang, A.C. Bovik, H.R. Sheikh, E.P. Simoncelli. Image quality assessment: from error visibility to structural similarity. IEEE Transactions on Image Processing, 12(4):600-612, Apr. 2006.

[11] J. Tian, Reversible data embedding using a difference expansion, IEEE Transaction on Circuits and Systems for Video Technology, 13(8), 2003. 